\title{
Ischemic Stroke in a Young Man: Unraveling the Domain of Myeloproliferative Disorders
}

\author{
Muhammad Ammar B. Hamid ${ }^{1}$, Aasim S. Sehbai ${ }^{1}$, Shahan Tariq ${ }^{1}$ \\ 1. Hematology and Oncology, Alabama Cancer Care, Anniston, USA
}

Corresponding author: Shahan Tariq, shahan_93@yahoo.com

\begin{abstract}
Ischemic stroke is a rare phenomenon in young adults. A complete workup for hypercoagulable and myeloproliferative disorders is a cornerstone of evaluation. Essential thrombocytosis is a chronic myeloproliferative disorder that primarily involves platelets. It may remain undiagnosed in patients and subsequently present in the form of ischemic stroke. The management of this disorder is complex and often involves cytoreduction therapies. The initiation of these drugs in such patients may lead to unnecessary adverse effects and complications. This case report is an attempt to highlight an underappreciated cause of stroke when assessing young individuals.
\end{abstract}

Review began 07/08/2021 Review ended 07/11/2021 Published 07/19/2021

() Copyright 2021

Hamid et al. This is an open access article distributed under the terms of the Creative Commons Attribution License CC-BY 4.0., which permits unrestricted use, distribution, and reproduction in any medium, provided the original author and source are credited.
Categories: Internal Medicine, Oncology, Hematology

Keywords: stroke, myeloproliferative disorder, essential thrombocythemia, mthfr mutation, stroke in young adults

\section{Introduction}

Thrombocythemia often develops as a secondary response in reaction to a number of states including chronic inflammation, malignancy, infection, iron deficiency, trauma, or surgery, and is mostly diagnosed as an incidental finding. Although very infrequent, it may occur due to an underlying process related to cellular replication and proliferation. These uncommon conditions are classified under the heading of myeloproliferative disorders (MPDs) as essential thrombocythemia (ET), chronic myeloid leukemia (CML), scarring agnogenic myeloid metaplasia (AMM), and polycythemia vera (PV). Furthermore, mutations in the thrombopoietin gene can result in the occasional occurrence of familial thrombocythemia [1]. Through this case, we have attempted to highlight an uncommon cause of ischemic stroke in young individuals.

\section{Case Presentation}

A 25-year-old man presented to the hematology/oncology clinic for a follow-up appointment. One week ago, the patient was admitted to the hospital with mild confusion and sudden onset weakness of the left side of his body. Motor strength was 2/5 in both the left upper and lower limbs. Babinski reflex could be appreciated in the left foot. An MRI of the brain (Figure 1) revealed an infarct lesion in the right parietaloccipital region consistent with a diagnosis of ischemic stroke. He was subsequently treated with tissue plasminogen activator (tPA) and later started on aspirin along with clopidogrel. Additional workup revealed an extremely high platelet count of $1030 \times 10^{9} / \mathrm{L}$. The rest of the cell counts were normal. Due to the unusual nature of this presentation in a young individual, a detailed workup for hypercoagulable and myeloproliferative disorders was initiated. The patient's stroke symptoms improved over the next five days and he was eventually discharged. 


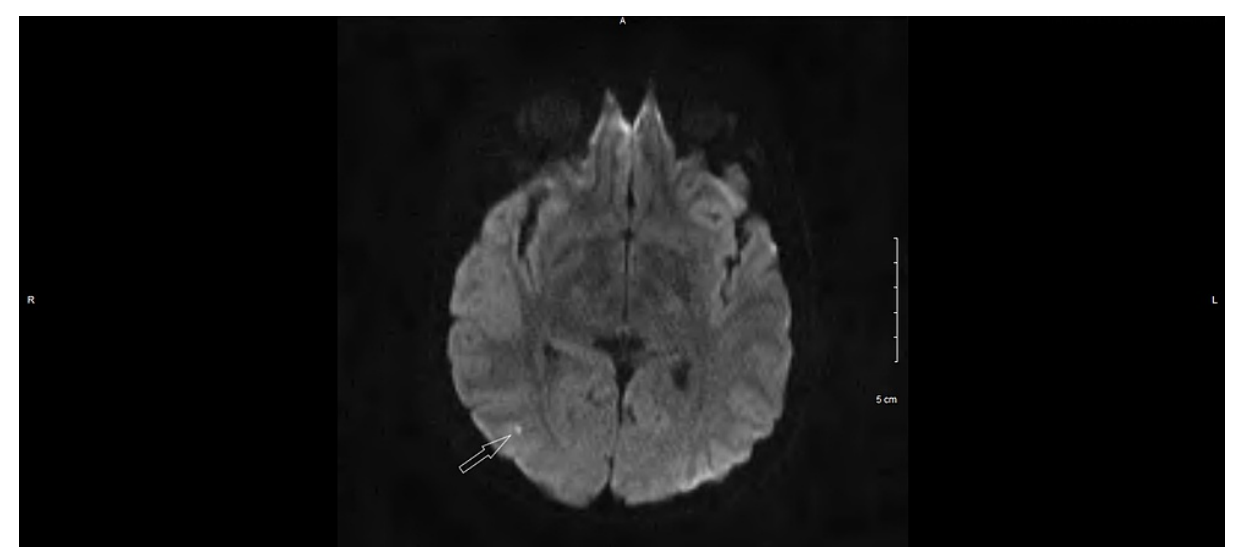

\section{FIGURE 1: MRI of the brain showing a hyperintense lesion in the right parietal-occipital region}

He complained of a mild headache, fatigue, and blurry vision since being discharged. He was vitally stable with cardiovascular and respiratory examination being normal. The abdomen was soft, non-tender, bowel sounds were positive and no evidence of hepato-splenomegaly was found. His labs revealed hemoglobin $(\mathrm{Hb})$ of $14.5 \mathrm{~g} / \mathrm{dL}$, white blood cell (WBC) count of $10.7 \times 10^{9} / \mathrm{L}$, and platelet count of $952 \times 10^{9} / \mathrm{L}$. His serum iron, total iron-binding capacity (TIBC), Vitamin B12, folate, and homocysteine levels were within the physiological range. Janus kinase 2 (JAK2) and methylenetetrahydrofolate reductase (MTHFR) heterozygous A1298C mutations were positive. The rest of the investigations including prothrombin time/partial thromboplastin time (PT/PTT), protein C, protein S, factor V, prothrombin gene mutation, anti-nuclear antibody (ANA), human immunodeficiency virus serology (HIV), rapid plasma reagent (RPR), lupus anticoagulant, anti-cardiolipin, and $\beta-2$ microglobulin antibodies were negative. Abdominal and duplex ultrasound of lower extremities were unremarkable. His past surgical history was positive for a $5.5 \mathrm{~cm}$ lipoma removal from his right shoulder. His past medical history did not reveal any significant findings. There was no history of any vitamin/mineral deficiency. No history of similar diseases in the family could be illustrated. He is a non-smoker, non-addict with no known drug allergies. He drinks alcohol socially.

A bone marrow biopsy showed hypercellularity and atypical megakaryocytic hyperplasia consistent with the diagnosis of essential thrombocytosis. Given his established history of thrombosis, he was advised to continue aspirin. Due to his young age, cytoreduction therapy with pegylated interferon-alpha (PEG-IFN- $\alpha$ ) is being started at a low dose of $45 \mu \mathrm{g}$ weekly and will be increased as required. From next week, hydroxyurea would be added to help while the effects of interferon kick in. He will be monitored with regular follow-up every four weeks.

\section{Discussion}

Out of all the myeloproliferative disorders, CML is the only one characterized by a specific mutation in the BCR-ABL arrangement. The rest of the MPDs are known as BCR-ABL negative. The presence of persistent non-reactive thrombocythemia usually leads to the diagnosis of ET. The main features observed in ET are a significant rise in the number of platelets and megakaryocytes. The definite origin of ET can perhaps be associated with several underlying factors in various patient subsets. However, its pathophysiology apparently seems to be related to an acquired interference in the signaling pathway involving thrombopoiesis. JAK2 mutation is a guanine to thymine somatic mutation at nucleotide 1849 in exon 14 and occurs when a gain of function substitute replaces valine with phenylalanine at position 617 . Genetic mutation for JAK2 has been found in a considerable number of patients suffering from either one of the three non-BCR-ABL MPDs i.e. PV, AMM, and ET [2,3], thereby essentially narrowing down the differentials by ruling out CML. Out of these, $23 \%$ to $57 \%$ of the patients having ET were also reported to have a mutation in JAK2 [4].

Differentiating between reactive and clonal thrombocythemia requires quite a number of investigations to be done which, no matter how laborious, are decisive in narrowing down the definite diagnosis whilst ruling out others as well as crucial in regard to employing the correct treatment protocols.

Although a large proportion of patients remain asymptomatic for a considerable duration, nearly $40 \%$ to almost $90 \%$ of patients suffering from ET show symptoms such as hemorrhage, thrombosis, and/or vasomotor disturbance when they ultimately present [5]. Thrombosis is three times more likely to occur in the arterial vasculature compared to the venous bed [6]. Around 30\% to $40 \%$ of patients present with platelet-mediated vasomotor symptoms including lightheadedness, headaches, migraine-like symptoms accompanied by visual disturbances which are transient in nature, focal neurological deficits, and syncope $[7,8]$. 
In a study, the risk of thrombotic events such as stroke, myocardial infarction, deep vein thrombosis, transient ischemic attacks, pulmonary embolism, retinal artery/vein occlusion, thrombosis of portal/hepatic veins, and limb/digital ischemia in those having ET was calculated. It was found to be about $6.6 \%$ per patient annually compared to the control population, which had a risk of only $1.2 \%$ per patient annually [9]. JAK2V617F mutation is present in approximately $50 \%$ to $60 \%$ of ET cases. It doubles the risk of thrombosis along with hypertension and smoking being substantial risk factors [10].

A retrospective chart review study involving patients having rare MTHFR polymorphisms concluded that there exists an increased risk of venous thromboembolism (VTE) associated with heterozygous/homozygous MTHFR variants. Meanwhile, hyperhomocysteinemia does not have a significant correlation with MTHFR polymorphisms. Therefore, assessment of VTE risk can be better estimated using genotyping of MTHFR [11]. Furthermore, the co-existence of ET and MTHFR mutation with hyperhomocysteinemia has been observed to result in an exponential increase with regards to the risk of occurrence of an ischemic phenomenon [12]. Our patient was a unique case having a mutation in MTHFR but normal homocysteine levels, an extremely rare entity.

Patients suffering from ET who have had an ischemic stroke are at higher risk for re-thrombosis and thus, they are additionally managed with cytoreductive agents along with antithrombotic medication. Given the abnormal production of platelets, there occurs only a sub-optimal suppression of platelets using the standard and recommended regimen of aspirin. Hence, to achieve ideal results, low-dose aspirin should be administered twice daily in an arterial thrombotic disease where the following risk factors are present: age of 60 and above, JAK2 mutation, and cardiovascular risk factors [13].

Treatment of ET revolves around the estimation of the relative risk of thrombosis and further transformation of disease. The risk of ET transforming to leukemia or other MPDs has been observed to increase over time [14]. Those above 60 years of age or having past history of thrombotic episodes are considered as high risk and should receive treatment with hydroxyurea, a cytoreductive agent. With regards to the treatment of thrombocythemia in ET, several RCTs have established the efficacy of hydroxyurea both when administered in comparison to a control as well as in combination therapy either with aspirin alone or anagrelide alongside $[15,16]$. IFN- $\alpha$ is also considered to be a reliable treatment, especially in pregnant or those who cannot tolerate hydroxyurea. IFN- $\alpha$ has the added benefit of resulting in a complete molecular response (CMR) where the JAK2-V617F becomes undetectable, although in fewer patients, alongside a complete hematologic response (CHR). Genotypic background may determine the ultimate clinical and molecular response to PEG-IFN- $\alpha$; however, it is worthy of mention that patients who did not have other mutations outside the JAK2 signal transducer and transcription activator pathway were more likely to achieve CMR [17].

Extreme thrombocytosis is defined as a platelet count of more than or equal to $1,000 \times 10^{9} / \mathrm{L}$ and such states are rarely observed. A retrospective study involving 535 patients having extreme thrombocytosis showed that only $2 \%$ were suffering from ET [18]. The initial presentation of our patient with ischemic stroke at the age of 25 and his subsequent diagnosis of ET along with a JAK2 mutation is no less than a rare occurrence. Hence, physicians should be mindful of the fact that there may exist underlying genetic predisposition in a patient of stroke or vice versa.

\section{Conclusions}

It is important to consider essential thrombocytosis as a potential cause of ischemic stroke when dealing with a young adult. Although there is a need for further studies in order to estimate and increase the effectiveness and safety of all available treatment modalities, it must ultimately be intended for coping with all known modifiable risk factors and thereby, reduce them. This objective becomes of utmost importance when the patient is young, such as ours, who potentially has decades to deal with the side effects of treatment as well as accumulate additional risk factors.

\section{Additional Information \\ Disclosures}

Human subjects: Consent was obtained or waived by all participants in this study. Conflicts of interest: In compliance with the ICMJE uniform disclosure form, all authors declare the following: Payment/services info: All authors have declared that no financial support was received from any organization for the submitted work. Financial relationships: All authors have declared that they have no financial relationships at present or within the previous three years with any organizations that might have an interest in the submitted work. Other relationships: All authors have declared that there are no other relationships or activities that could appear to have influenced the submitted work.

\section{References}

1. Wiestner A, Schlemper RJ, van der Maas AP, Skoda RC: An activating splice donor mutation in the thrombopoietin gene causes hereditary thrombocythaemia. Nat Genet. 1998, 18:49-52. 10.1038/ng0198-49 
2. James C, Ugo V, Le Couédic JP, et al.: A unique clonal JAK2 mutation leading to constitutive signalling causes polycythaemia vera. Nature. 2005, 434:1144-8. 10.1038/nature03546

3. Kralovics R, Passamonti F, Buser AS, et al.: A gain-of-function mutation of JAK2 in myeloproliferative disorders. N Engl J Med. 2005, 352:1779-90. 10.1056/NEJMoa051113

4. Tefferi A, Gilliland DG: The JAK2V617F tyrosine kinase mutation in myeloproliferative disorders: status report and immediate implications for disease classification and diagnosis. Mayo Clin Proc. 2005, 80:947-58. 10.4065/80.7.947

5. Harrison CN, Green AR: Essential thrombocythemia. Hematol Oncol Clin North Am. 2003, 17:1175-1190. 10.1016/s0889-8588(03)00082-0

6. Blann A, Caine G, Bareford D: Abnormal vascular, platelet and coagulation markers in primary thrombocythaemia are not reversed by treatments that reduce the platelet count. Platelets. 2004, 15:447-9. 10.1080/09537100412331272541

7. Fenaux P, Simon M, Caulier MT, Lai JL, Goudemand J, Bauters F: Clinical course of essential thrombocythemia in 147 cases. Cancer. 1990, 66:549-556. 10.1002/1097-0142(19900801)66:3<549::aidcncr2820660324>3.0.c0;2-6

8. Kesler A, Ellis MH, Manor Y, Gadoth N, Lishner M: Neurological complications of essential thrombocytosis (ET). Acta Neurol Scand. 2000, 102:299-302. 10.1034/j.1600-0404.2000.102005299.x

9. Cortelazzo S, Viero P, Finazzi G, D'Emilio A, Rodeghiero F, Barbui T: Incidence and risk factors for thrombotic complications in a historical cohort of 100 patients with essential thrombocythemia. J Clin Oncol. 1990, 8:556-62. 10.1200/JCO.1990.8.3.556

10. Khaheshi I, Memaryan M, Taherkhani M, Serati A, Movahed MR: Acute ST-segment elevation myocardial infarction as the first manifestation of essential thrombocytosis successfully treated with thrombectomy alone. Cardiovasc Interv Ther. 2016, 31:275-8. 10.1007/s12928-015-0347-z

11. Liu F, Silva D, Malone MV, Seetharaman K: MTHFR A1298C and C677T polymorphisms are associated with increased risk of venous thromboembolism: a retrospective chart review study. Acta Haematol. 2017, 138:208-15. 10.1159/000480447

12. Pereira M, Morais SA, Pereira N, Lima AL, Barreto V: Splenic infarcts-when the cause is multifactorial: MTHF gene mutation and essential thrombocytosis. Galicia Clin. 2016, 77:75-7. 10.22546/36/843

13. Das S, Deb A: Antithrombotic management in ischemic stroke with essential thrombocythemia: current evidence and dilemmas (IN PRESS). Med Princ Pract. 2021, 10.1159/000516471

14. Wolanskyj AP, Schwager SM, McClure RF, Larson DR, Tefferi A: Essential thrombocythemia beyond the first decade: life expectancy, long-term complication rates, and prognostic factors. Mayo Clin Proc. 2006, 81:159-66. 10.4065/81.2.159

15. Cortelazzo S, Finazzi G, Ruggeri M, Vestri O, Galli M, Rodeghiero F, Barbui T: Hydroxyurea for patients with essential thrombocythemia and a high risk of thrombosis. N Engl J Med. 1995, 332:1132-6. 10.1056/NEJM199504273321704

16. Harrison CN, Campbell PJ, Buck G, et al.: Hydroxyurea compared with anagrelide in high-risk essential thrombocythemia. N Engl J Med. 2005, 353:33-45. 10.1056/NEJMoa043800

17. Quintás-Cardama A, Abdel-Wahab O, Manshouri T, et al.: Molecular analysis of patients with polycythemia vera or essential thrombocythemia receiving pegylated interferon $\alpha-2 a$. Blood. 2013, 122:893-901. 10.1182/blood-2012-07-442012

18. Wiwanitkit V: Extreme thrombocytosis: what are the etiologies? . Clin Appl Thromb Hemost. 2006, 12:85-7. $10.1177 / 107602960601200113$ 